\title{
Simplified Processing for High Efficiency Wireless Communication Employing Multiple Antenna Array
}

\author{
S.Taruna \\ Computer Science \\ Department \\ Banasthali University \\ Jaipur, India
}

\author{
Ikpreet Kaur \\ Information Technology \\ Department \\ Banasthali University \\ Jaipur, India
}

\author{
Bhumika Pahwa \\ Information Technology \\ Department \\ Banasthali University \\ Jaipur, India
}

\begin{abstract}
:
The use of multiple antennas for wireless systems has gained vast interest during the last decade - both in academic world and industry. Multiple antennas can be utilized in order to accomplish a multiplexing gain, a diversity gain, or an antenna gain, thus enhancing the bit rate, the error performance, or the signal-to-noise-plusinterference ratio of wireless systems, respectively. With the huge amount of research in the field of multipleantenna systems, often called multiple-input multipleoutput (MIMO) systems, has evolved rapidly. This paper is intended to provide the complete understanding of the MIMO System. In this work, the performance of MIMO Systems have been analyzed with transmit and receive diversity. This paper also includes the comparison of MIMO technology with SISO, MISO and SIMO on the basis of capacity by simulating different scenarios in MATLAB.
\end{abstract}

\section{Keywords:}

MIMO, SISO, Spatial Multiplexing, Spatial Diversity

\section{INTRODUCTION}

Physical limitations of the wireless network provide a technical challenge for reliable wireless communication systems. Techniques that improve spectral efficiency and overcome various channel impairments such as signal fading and interference have made a huge contribution to the growth of wireless communications. Moreover, the need for high-speed wireless Internet has led to the demand for technologies delivering higher capacities and link reliability than achieved by current systems [1].

The development of communication system begins with Single Input and Single Output (SISO), Single Input and Multiple Output (SIMO), and then followed by Multiple Input and Single Output (MISO) and the recent communication system technique is Multiple Input and Multiple Output (MIMO) [2].

MIMO technology constitutes a breakthrough in wireless communication system design. MIMO was proposed in 1993 Agogyaswami Paulraj and Thomas Kailath. In 1996, Greg Greg Raleigh and Gerard J. Foschini introduce new approaches to MIMO technology to refine or to improve the link throughput effectively using multiple antennas at both transmitter and receiver end [3].

MIMO structure consist of multiple antennas at both transmitter and receiver end for improving the communication performance. The technology offers a number of benefits that helps to meet the challenges posed by both the impairments in the wireless channel as well as resource constraints.

The scheme of using multiple antenna configuration instead of a single one has proven to be successful in enhancing data transfer rate, coverage, and overall performance of radio networks. The increase in spectral in efficiency offered by MIMO Systems is based on the utilization of space (or antenna) diversity at both the transmitter and the receiver. Due to the utilization of space diversity, MIMO systems are also referred to as multiple-element antenna systems (MEAs). MIMO technology provides various benefits such as array gain, spatial diversity gain, spatial multiplexing gain, and interference reduction and avoidance [4].

The benefits of MIMO lead many to believe that it is the most promising of new developing wireless technologies. The table below provides the data of the world top five countries with highest of internet users in 2012 [5].

Table1. Number of internet users in different countries

\begin{tabular}{|l|l|l|l|l|}
\hline S.No & Country & $\begin{array}{l}\text { Population, } \\
2012 \text { Est }\end{array}$ & $\begin{array}{l}\text { Internet } \\
\text { users } \\
2000\end{array}$ & $\begin{array}{l}\text { Internet } \\
\text { users } \\
2102\end{array}$ \\
\hline 1 & China & $1,343,239,923$ & $22,500,000$ & $538,000,000$ \\
\hline 2 & America & $313,847,465$ & $95,354,000$ & $245,203,319$ \\
\hline 3 & India & $1,205,073,612$ & $5,000,000$ & $137,000,000$ \\
\hline 4 & Japan & $127,368,088$ & $47,080,000$ & $101,228,736$ \\
\hline 5 & Brazil & $193,946,886$ & $5,000,000$ & $88,494,756$ \\
\hline
\end{tabular}

Thus the use of MIMO technology is highly useful for the growing number of internet users as it increases the capacity of the wireless network.

The key benefits of multiple antennas for wireless communication are described below:

\subsection{Higher Bit Rates}

In multiple antenna system with $M$ transmit antennas; the bit rate is enhanced by a factor of $M$ without increasing the bandwidth or transmission power. The gain in bit rate when compared to single antenna system is called multiplexing gain [6].

\subsection{Smaller Error Rates}

Multiple Input Multiple Output techniques can be used to improve the error rate of the wireless network, by transmitting the redundant signals to the receiver. Space time coding is a two dimensional coding in time and 
space, where information signals is spread out over multiple antennas [6].

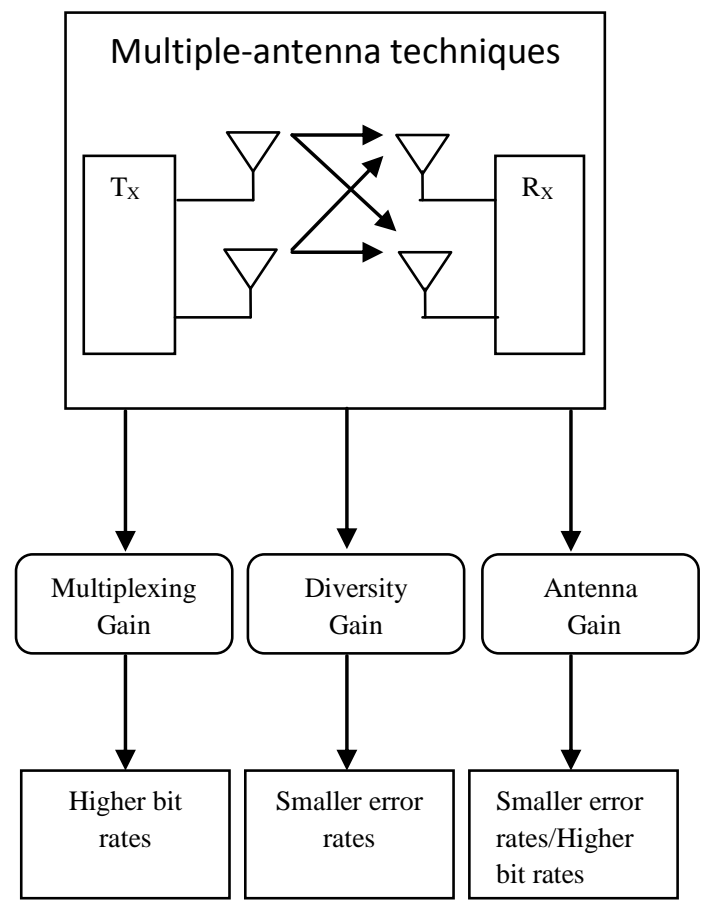

Fig 1: Benefits of MIMO in wireless communication

\subsection{Improved Signal-to-noise ratio}

Multiple antenna technique can be used to increase the signal-to-noise ratio (SNR) at the destination end. The improved value of SNR can be achieved by a technique called adaptive antenna array or smart antennas [7].

\section{SPATIAL MUTIPLEXING}

In multiple antenna system with $\mathrm{M}$ transmit and $\mathrm{N}$ receive antenna, capacity grows linearly with the minimum of $\mathrm{M}$ and $\mathrm{N}$ without requiring extra bandwidth or transmission power. The basic structure of spatial multiplexing scheme consists of transmitter, information sequence and receiver. At the transmitter, the information bit sequence is split into $\mathbf{M}$ sub-sequences, that are modulated and transmitted simultaneously over the transmit antennas using the same frequency band. [8].

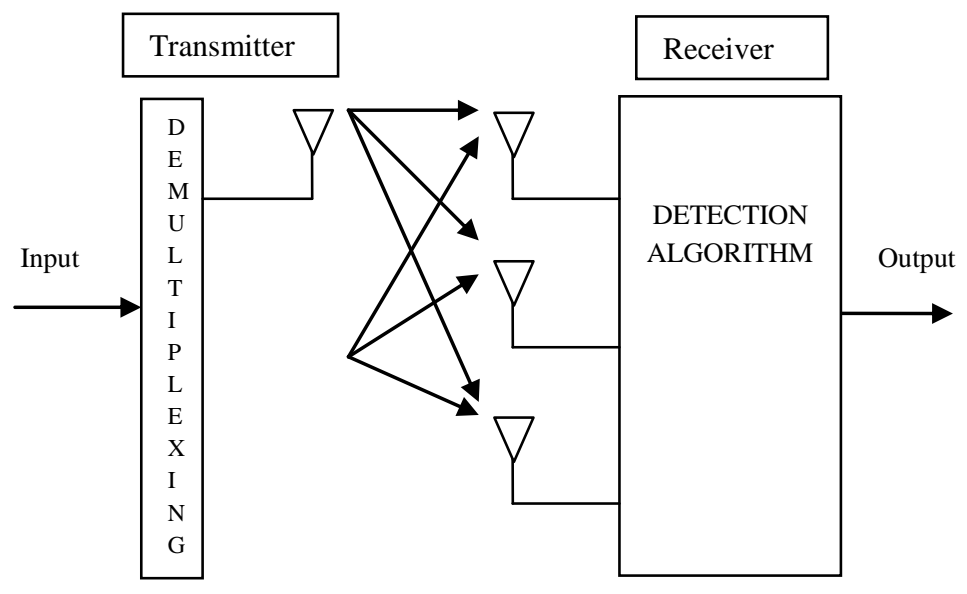

Fig 2: Structure of Spatial Multiplexing

3. SPATIAL DIVERSITY TECHNIQUES

In contrast to spatial multiplexing techniques, where the main objective is to provide higher bit rates compared to a singleantenna system, spatial diversity techniques predominantly aim at an improved error performance. This is accomplished on the basis of a diversity gain and a coding gain. Indirectly, spatial diversity techniques can also be used to enhance bit rates, when employed in conjunction with an adaptive modulation/channel coding-scheme. There are two types of spatial diversity, referred to as macroscopic and microscopic diversity. Macroscopic diversity is related with shadowing effects in wireless communication scenarios, due to major obstacles between transmitter and receiver. Macroscopic diversity can be gained if there are multiple transmit or receive antennas, that are spatially separated on a large scale. In this case, the probability that all links are simultaneously obstructed is smaller than that for a single

link.

Microscopic diversity is available in rich scattering environments with multipath fading. Microscopic diversity can be gained by employing multiple co-located antennas. Similar to macroscopic diversity, the diversity gains are due to the fact that the probability of all links being simultaneously in deep fades decreases with the number of antennas used [9].

\section{CHANNEL CAPACITY FOR DIFFERENT ANTENNA MODEL}

Channel capacity was established by Claude Shannon in 1940 s, by using the mathematical theory of communication. The capacity of a channel is denoted by $\mathrm{C}$. The channel capacity $\mathrm{C}$ is the maximum rate at which reliable communication can be performed, without any constraints on transmitter and receiver complexity.

\subsection{Channel Capacity of SISO}

The simplest form of radio link can be defined in MIMO terms as SISO. This is effectively a standard radio channelthis transmitter operates with one antenna as does the receiver.

There is no diversity and no additional processing required. Equation (1) shows the channel capacity of SISO System:

$$
C=\log _{2}(1+\gamma) b p s / H z
$$




\subsection{Channel Capacity of MISO}

MISO system is an antenna system in which we have multiple transmitting antennas and one receiving antenna. The MISO channel model provides transmits diversity because of multiple numbers of antennas at transmitter side, and slow logarithmic rise of capacity with increasing number of antennas. Equation (2) shows the channel capacity of MISO System:

$$
C=\log _{2}(1+N \gamma) b p s / H z
$$

Where, $\mathrm{N}$ is the number of transmitting antennas.

\subsection{Channel Capacity of SIMO}

The channel capacity of SIMO systems provides receiver diversity because of multiple antennas at receiver side and capacity is slow logarithmic rising with increasing number of antennas. Equation (3) shows the channel capacity of SIMO System:

$$
C=\log _{2}(1+M \gamma) b p s / H z
$$

Where, $\mathrm{M}$ is the number of receiving antenna.

\subsection{Channel Capacity of MIMO}

MIMO system has multiple antennas on both sides either on receiving or transmitting side. It is also in the category of smart antennas that is used to improve the communication performance. Equation (4) shows the channel capacity of MIMO:

$$
C=\log _{2}(1+N M \gamma) b p s / H z
$$

Where, $\mathrm{N}$ is the number of transmitting antenna and $\mathrm{M}$ is the number of receiving antenna.

\section{RESULT AND SIMULATION}

In this paper, the multiple antenna technology has been compared with other antenna technology such as SISO, MISO and SIMO by plotting a capacity versus number of antenna graph and keeping the value of SNR constant. This section is divided into four cases. In each case the graph has been plotted for MIMO System with $12 \times 12$ antenna configuration and different values of SNR such as $0 \mathrm{db}, 10 \mathrm{db}, 30 \mathrm{db}$ and $50 \mathrm{db}$.

\subsection{Case1: Analysis of MIMO System when SNR is $0 \mathrm{db}$}

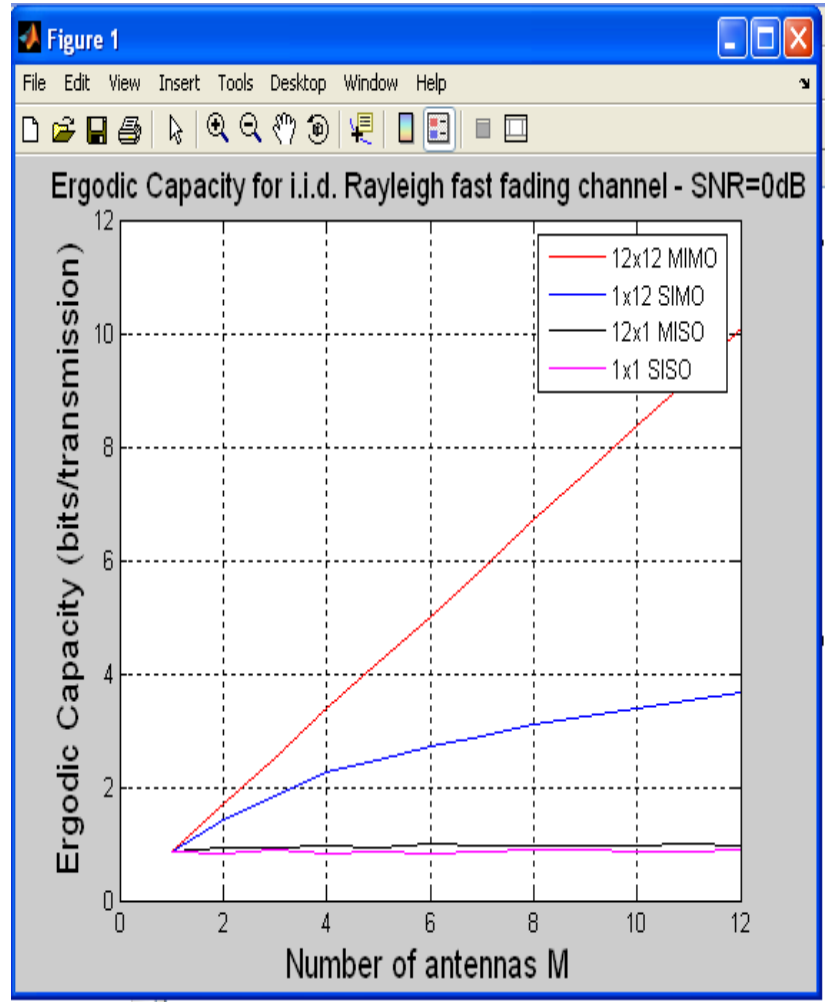

Fig 3: Capacity versus No. of Antenna when SNR is Odb

Table 1: Capacity Analysis of MIMO System when SNR is Odb

\begin{tabular}{|l|l|l|l|l|l|}
\hline S.NO & \multirow{2}{*}{\begin{tabular}{c} 
No. of \\
\multirow{nnyyyy}{*}{ Antennas }
\end{tabular}} & \multicolumn{4}{|c|}{ Capacity(bps/Hz) } \\
\cline { 3 - 6 } & & MIMO & SIMO & MISO & SISO \\
\hline 1 & 1 & 0.88 & 0.87 & 0.84 & 0.88 \\
\hline 2 & 2 & 1.67 & 1.44 & 0.90 & 0.86 \\
\hline 3 & 4 & 3.31 & 2.21 & 0.94 & 0.83 \\
\hline 4 & 6 & 4.19 & 2.75 & 0.96 & 0.85 \\
\hline 5 & 8 & 5.88 & 3.12 & 0.97 & 0.84 \\
\hline 6 & 10 & 7.51 & 2.41 & 0.98 & 0.83 \\
\hline 7 & 12 & 10.04 & 3.63 & 0.99 & 0.82 \\
\hline
\end{tabular}




\subsection{Case2: Analysis of MIMO System when SNR is $10 \mathrm{db}$}

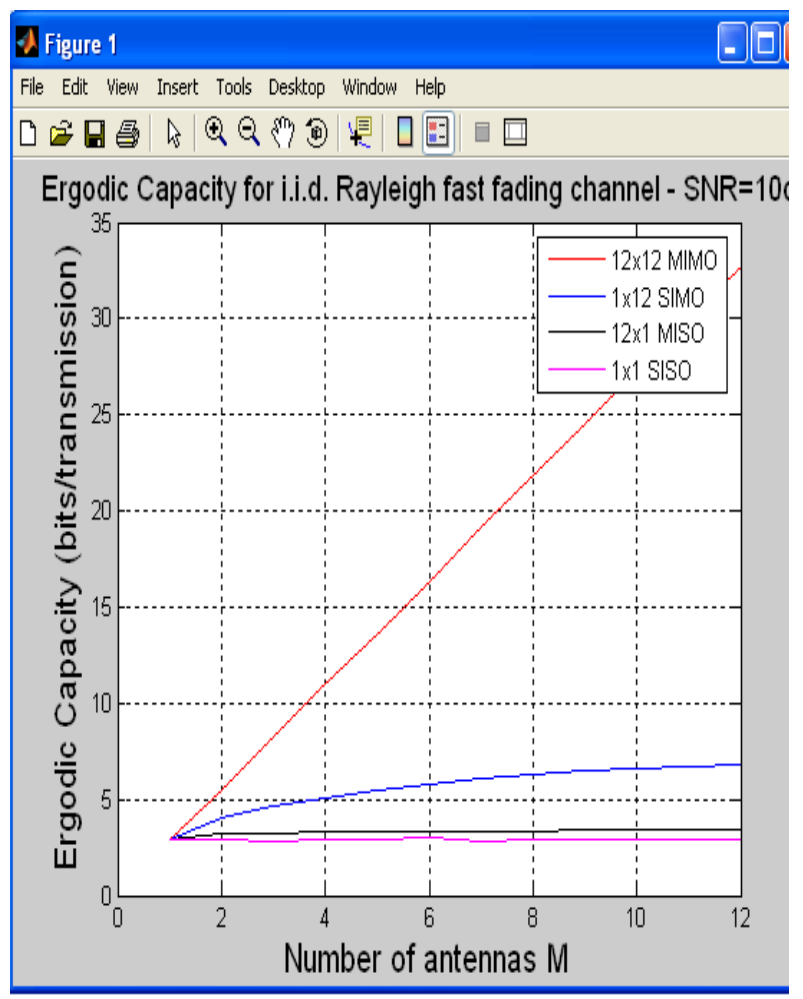

Fig 4: Capacity versus No. of Antenna when SNR is $10 \mathrm{db}$

Table 2: Capacity Analysis of MIMO System when

\begin{tabular}{|c|c|c|c|c|c|}
\hline \multicolumn{6}{|c|}{$\begin{array}{r}\text { SNR } \\
\text { is } 10 \mathrm{db}\end{array}$} \\
\hline \multirow{2}{*}{$\begin{array}{l}\text { S.N } \\
\text { O }\end{array}$} & \multirow{2}{*}{$\begin{array}{c}\text { No. of } \\
\text { Antenna } \\
\text { s }\end{array}$} & \multicolumn{4}{|c|}{ Capacity(bps/Hz) } \\
\hline & & $\begin{array}{l}\text { MIM } \\
\text { O }\end{array}$ & $\begin{array}{l}\text { SIM } \\
\text { O }\end{array}$ & $\begin{array}{l}\text { MIS } \\
\text { O }\end{array}$ & $\begin{array}{l}\text { SIS } \\
\mathrm{O}\end{array}$ \\
\hline 1 & 1 & 2.81 & 2.88 & 2.84 & 2.95 \\
\hline 2 & 2 & 5.50 & 4.02 & 3.22 & 2.86 \\
\hline 3 & 4 & 10.88 & 5.18 & 3.31 & 2.85 \\
\hline 4 & 6 & 16.30 & 5.83 & 3.35 & 2.92 \\
\hline 5 & 8 & 21.74 & 6.24 & 3.40 & 2.93 \\
\hline 6 & 10 & 27.22 & 6.55 & 3.37 & 2.92 \\
\hline 7 & 12 & 32.65 & 6.87 & 4.41 & 2.94 \\
\hline
\end{tabular}

\subsection{Case3: Analysis of MIMO System when SNR is $30 \mathrm{db}$}

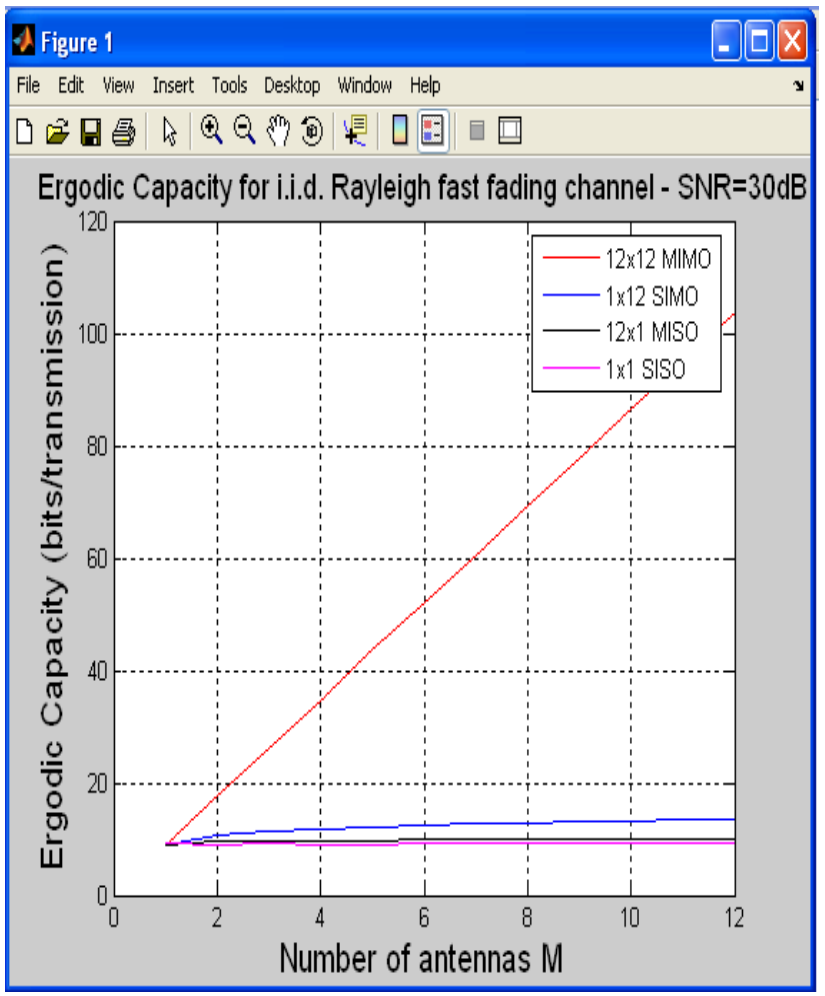

Fig 5: Capacity versus No. of Antenna when SNR is 30db

Table 3: Capacity Analysis of MIMO System when SNR is $30 \mathrm{db}$

\begin{tabular}{|l|l|l|l|l|l|}
\hline \multirow{2}{*}{ S.NO } & \multirow{2}{*}{$\begin{array}{c}\text { No. of } \\
\text { Antennas }\end{array}$} & \multicolumn{4}{|c|}{ Capacity(bps/Hz) } \\
\cline { 3 - 6 } & & MIMO & SIMO & MISO & SISO \\
\hline 1 & 1 & 9.81 & 9.18 & 9.13 & 9.07 \\
\hline 2 & 2 & 26.22 & 10.55 & 9.65 & 9.18 \\
\hline 3 & 4 & 34.87 & 11.70 & 9.75 & 9.11 \\
\hline 4 & 6 & 51.89 & 12.39 & 9.82 & 9.26 \\
\hline 5 & 8 & 69.79 & 12.88 & 9.86 & 9.15 \\
\hline 6 & 10 & 86.39 & 13.18 & 9.89 & 9.17 \\
\hline 7 & 12 & 103.57 & 13.47 & 9.90 & 9.18 \\
\hline
\end{tabular}




\subsection{Case4: Analysis of MIMO System when SNR is $50 \mathrm{db}$}

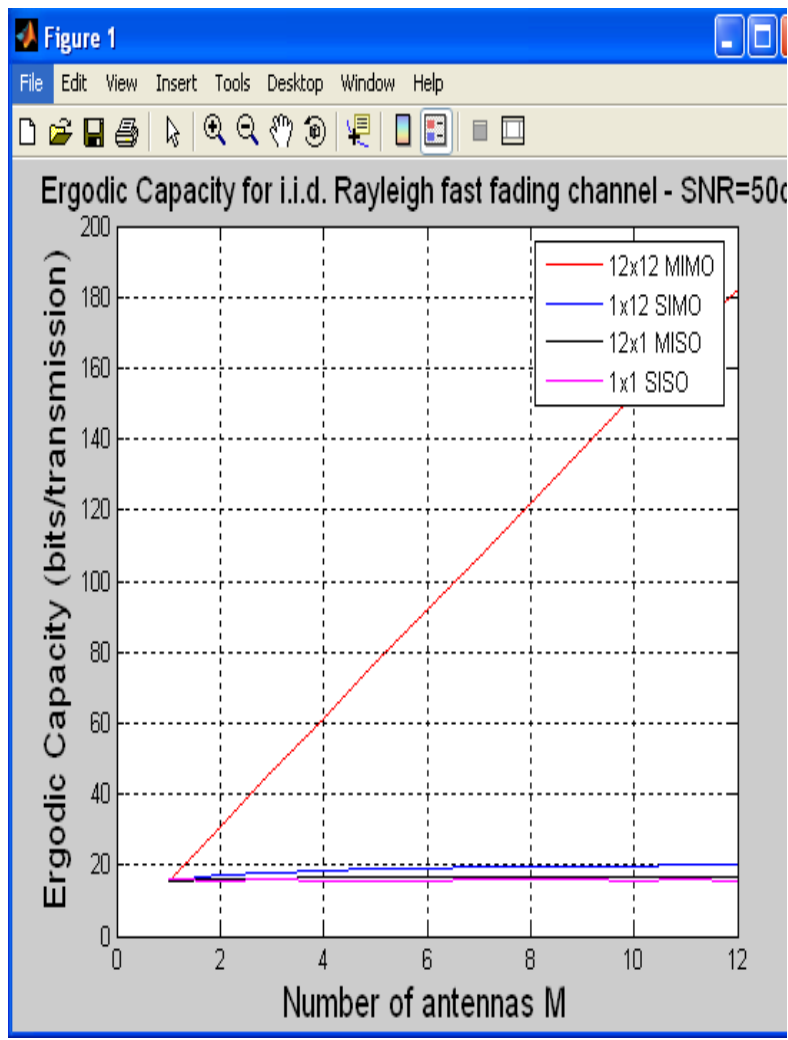

Fig 6: Capacity versus No. of Antenna when SNR is 50db

Table 4: Capacity Analysis of MIMO System when SNR

Is 50db

\begin{tabular}{|l|l|l|l|l|l|}
\hline S.NO & \multirow{2}{*}{\begin{tabular}{c} 
No. of \\
\cline { 3 - 6 }
\end{tabular}} & Antennas & \multicolumn{4}{|c|}{ Capacity(bps/Hz) } \\
\cline { 3 - 6 } & & MIMO & SIMO & MISO & SISO \\
\hline 1 & 1 & 15.70 & 15.79 & 15.68 & 15.80 \\
\hline 2 & 2 & 31.07 & 17.16 & 16.28 & 15.68 \\
\hline 3 & 4 & 61.54 & 18.50 & 16.43 & 15.72 \\
\hline 4 & 6 & 91.67 & 19.06 & 16.51 & 15.75 \\
\hline 5 & 8 & 121.98 & 19.51 & 16.47 & 15.90 \\
\hline 6 & 10 & 152.48 & 19.83 & 16.53 & 15.72 \\
\hline 7 & 12 & 182.69 & 20.12 & 16.54 & 12.92 \\
\hline
\end{tabular}

\section{CONCLUSION}

The rapidly growing demand for wireless data traffic poses the challenge of how to increase the capacity of the wireless system without raising the transmitted power of the transmitter. In this paper we have presented an introduction to an upcoming technology called MIMO. In this technology more than one antenna is used at the transmitter as well as at the receiver side which increases the capacity of the wireless system noticeably. In above section we have analyzed MIMO Systems with different antenna configuration and have also compared these systems with other technologies such as SISO, MISO and MISO. After analyzing the results we can conclude that for MIMO Systems the ergodic capacity increases drastically with the increase in number of antennas but for other technologies such as SIMO and MISO the capacity increases very slightly with increase in number of antennas. This paper also includes the numerical results for the same.

\section{REFERENCES}

[1] J.Aparna "Fundamental Overview and Simulation of Space-Time Coded MIMO System" May 2005

[2] Nur Izzati Binti Mohd Ali " Characterization of Wireless Channel by Using Angle Diversity" May 2011

[3] Sugandha Agarwal, Prabhjyot Kaur, “ Characteristics Parameters Evaluation for MIMO Based Communication System", International Conference on Computing Communication System and Informatics Management, July2012

[4] Amitabha Das, Hung Keng Pung, Francis Bu Sung Lee, Lawrence Wai Choong Womg (Eds)," Networking 2008 Ad-hoc and Sensor Networks Wireless Networks, Next Generation Internet", 7thInternational IFIP_TC6 Networking Conference, Singapore, May 2008, Proceedings

[5] www.internetworldstats.com/top20.htm

[6] Jan Mietzner, Robert Schober, Lutz Lampe, Wolfgang H. Gerstacker, Peter A. Hoeher "Multiple-Antenna Techniques for Wireless Communications - A Comprehensive Literature Survey" IEEE Communications Surveys \& Tutorials, Vol. 11, no. 2, Second Quarter 2009

[7] L. C. Godara, "Application of antenna arrays to mobile communications- Part I: Performance improvement, feasibility, and system considerations; Part II: Beam-forming and direction-of-arrival considerations," Proc. IEEE, vol. 85, no. 7/8, pp. 1031-1060, 1195-1245, July/Aug. 1997.

[8] E. Telatar, "Capacity of multi-antenna Gaussian channels," Europ. Trans. Telecommun., vol. 10, no. 6, pp. 585-595, Nov./Dec. 1999

[9] S. N. Diggavi, N. Al-Dhahir, A. Stamoulis, and A. R. Calderbank, "Great expectations: The value of spatial diversity in wireless networks," Proc. IEEE, vol. 92, no. 2, pp. 219-270, Feb. 2004 